\title{
Deformidade corneana em usuário de lente de contato portador de ceratocone: relato de caso
}

\author{
Corneal warpage in contact lens wearer with keratoconus: a case report
}

César Lipener ${ }^{(1)}$

Oftalmologista do Setor de Lentes de Contato do Departamento de Oftalmologia da Universidade Federal de São Paulo ( UNIFESP/EPM ).

Endereço para correspondência: Dr. César Lipener. Rua Manoel da Nóbrega, 595 conj. 91. São Paulo (SP), Brasil. CEP 04001-083. Tel/fax: 885-7019.

\begin{tabular}{|c|}
\hline RESUMO \\
\hline $\begin{array}{l}\text { Introdução: Alterações topográficas podem ocorrer secundaria- } \\
\text { mente ao uso de uso de lentes de contato duras ou gelatinosas. O } \\
\text { termo "corneal warpage" é utilizado para denominar as deformi- } \\
\text { dades corneanas causadas pelas lentes. O quadro topográfico da } \\
\text { fase inicial do ceratocone pode ser muito parecido com o de um } \\
\text { paciente com este quadro. } \\
\text { Objetivo: Mostrar um paciente portador de ceratocone, usuário de } \\
\text { lentes de contato rígida gás-permeável (RGP) que desenvolveu qua- } \\
\text { dro de "corneal warpage", diagnosticado e acompanhado por topo- } \\
\text { grafias e exames clínicos seriados. } \\
\text { Relato de caso: O paciente é um engenheiro de } 40 \text { anos com } \\
\text { diagnóstico de ceratocone bilateral há } 12 \text { anos, usando lentes RGP } \\
\text { desde então. No primeiro exame em } 5 / 95 \text {, a refração de OD foi } \\
\text { impossível de se determinar e a ceratometria era maior que } 60,00 \text { D. } \\
\text { Foi feita topografia, que se mostrou compatível com ceratocone, } \\
\text { tendo sido adaptada uma lente Soper, com visão de } 20 / 70 \text {. Após um } \\
\text { ano, uma topografia de controle mostrou aumento da curvatura } \\
\text { central e aplanamento da periferia inferior. O uso da lente foi } \\
\text { suspenso e após } 6 \text { meses houve regressão das mudanças, tendo sido } \\
\text { adaptadas novas lentes com melhor padrão e visão de } 20 / 40 \text {. } \\
\text { Discussão: O caso apresentado evidencia a ocorrência de deformi- } \\
\text { dade corneana em um portador de ceratocone usuário de lente RGP. } \\
\text { O autor discute a fisiopatologia e o diagnóstico clínico e topográfico } \\
\text { do "corneal warpage", lembrando que a exemplo de pacientes nor- } \\
\text { mais, os pacientes com ceratocone podem apresentar estas alterações, } \\
\text { que devem ser diferenciadas da própria evolução da doença. }\end{array}$ \\
\hline
\end{tabular}

Palavras-chave: Ceratocone; Lente de contato; Deformidade corneana.

\section{INTRODUÇ̃̃̃O}

Alterações topográficas da córnea podem ocorrer secundariamente ao uso diário ou prolongado de lentes de contato duras ou gelatinosas. O termo "corneal warpage" é utilizado para denominar as deformidades corneanas causadas pelas lentes de contato, reversíveis ou não e que não estejam relacionadas com edema de córnea ${ }^{1}$.

Esta complicação pode ocorrer quando a lente de contato interfere nas atividades fisiológicas normais da córnea ou por forças mecânicas resultantes da pressão exercida pelas pálpebras sobre as lentes e transferida para a córnea ${ }^{2}$. Alguns estudos sugerem que estas alterações ocorrem em pelo menos um dos meridianos corneanos em $70 \%$ dos usuários de lentes de contato ${ }^{3}$. 
Embora os portadores destas deformidades sejam freqüentemente assintomáticos, o paciente pode se queixar de visão borrada com óculos, injeção conjuntival, sensação de corpo estranho e diminuição no tempo de uso das lentes. Nos casos mais severos pode ocorrer perda da melhor acuidade visual corrigida com óculos, produção de marcas de compressão e deformação permanente da córnea ${ }^{4}$. Os sinais podem ainda incluir mudanças na refração e na ceratometria, quando comparados com os valores de antes da adaptação, além da distorção das miras ceratométricas ${ }^{1}$. O paciente típico é aquele usuário de longa data, satisfeito, sem queixas visuais e que geralmente não tem óculos ${ }^{2}$.

A topografia corneana computadorizada é um método eficiente e sensível para diagnóstico e acompanhamento das alterações corneanas em usuários de lente ${ }^{5,1}$. O quadro topográfico da fase inicial do ceratocone pode ser muito parecido com o de um paciente com "corneal warpage" 6 .

O ceratocone é um processo degenerativo da córnea que leva a seu afinamento e ectasia. $\mathrm{O}$ astigmatismo irregular e as cicatrizes são responsáveis pela baixa visão. Com etiologia ainda indeterminada, pode estar associado com atopia e retardo mental. A conduta depende da severidade do caso, mas o uso de lentes de contato ainda é o principal recurso óptico usado, principalmente nos casos mais avançados ${ }^{7}$. Alguns autores mencionam a possibilidade do uso de lentes causar um tipo específico de ceratocone ${ }^{8,9}$, porém há pouca referência quanto ao aparecimento de deformidade corneana induzida por lentes de contato em portadores desta doença.

\section{OBJETIVO}

O objetivo deste relato é mostrar um paciente portador de ceratocone, usuário de lente de contato rígida gás-permeável (RGP), que desenvolveu quadro de "corneal warpage", diagnosticado e acompanhado por topografias e exames clínicos seriados. O autor ressalta ainda a dificuldade de se fazer a distinção entre a deformidade corneana e a própria evolução da doença.

\section{RELATO DO CASO}

Trata-se de um paciente de 40 anos, engenheiro, com diagnóstico de ceratocone em ambos os olhos há 12 anos, usando lentes rígidas gás-permeáveis (RGP) desde então. $\mathrm{Na}$ primeira consulta (5/95) estava sem lentes há 2 dias. A refração foi impossível de ser determinada no olho direito e no olho esquerdo era $-5,00 \mathrm{DE}=-1,25 \mathrm{DC} 125 \mathrm{com}$ visão de $20 / 30$ neste olho. A ceratometria era maior que $60,00 \mathrm{D}$ nos dois meridianos de OD e em OE era 44,75/47,00 a 85, com miras irregulares em AO. O fundo de olho e a pressão intra-ocular eram normais e à biomicroscopia apresentava estrias de Vogt em ambos os olhos e um leucoma paracentral em OD. A topografia corneana computadorizada dos 2 olhos nesta data é apresentada nas figuras 1 e 2. Nesta ocasião, foram adaptadas lentes rígidas gás permeáveis (material RXD DK 45) em ambos os olhos com as seguintes características:

\begin{tabular}{lccccc}
\hline $\begin{array}{l}\text { Tipo de } \\
\text { lente }\end{array}$ & $\begin{array}{c}\text { curva } \\
\text { base }\end{array}$ & poder & diâmetro & visão & $\begin{array}{c}\text { posição da } \\
\text { lente }\end{array}$ \\
\hline OD Soper & $\begin{array}{c}56,00 / \\
45,00\end{array}$ & $-15,00$ & $8,5 \mathrm{~mm}$ & $20 / 70$ & $\begin{array}{c}\text { descentrada } \\
\text { inferior }\end{array}$ \\
OE esférica & 45,25 & $-6,25$ & $9,2 \mathrm{~mm}$ & $20 / 25$ & \begin{tabular}{c} 
centralizada \\
\hline
\end{tabular} \\
\hline
\end{tabular}

Em 6/96, um ano após a adaptação das lentes, retornou para controle de rotina e observou-se uma alteração importante no padrão topográfico da córnea, com aumento da curvatura central e aplanamento inferior. Diante deste quadro, foram feitas 3 hipóteses diagnósticas: 1) melhora da topografia pois a curva base da lente anterior era muito plana em relação à córnea; 2 ) piora do quadro pois a nova lente estava descentrada inferiormente; 3) evolução do ceratocone.

Frente a estas hipóteses, decidiu-se pela suspensão do uso da lente do OD com posterior seguimento clínico e topográfico. As figuras 3,4 e 5 mostram os exames topográficos seguintes, onde se nota a evolução das alterações corneanas.

Com o quadro aparentemente estabilizado, o exame clínico mostrou os seguintes dados no OD: Refração: $-18,00 \mathrm{DE}$ com AV 20/200 e ceratometria: 58,87 / 62,12 20 .

Foi adaptada nova lente Soper RGP com o mesmo material e com os seguintes parâmetros:

\begin{tabular}{ccccc}
\hline curva base & diâmetro & poder & visão & posição \\
\hline $57,00 / 45,00$ & $9,4 \mathrm{~mm}$ & $-14,00$ & AV 20/40 & centralizada \\
\hline
\end{tabular}

No último exame, em 11/97, o quadro estava inalterado, com o paciente usando a lente por 14 horas por dia, sem problemas.

\section{DISCUSSÃO}

O presente caso evidencia a ocorrência de deformidade corneana em um portador de ceratocone usuário de lente de contato, situação pouco descrita na literatura.

Há divergências em estudos publicados sobre "corneal warpage", já que alguns mostram aplanamento e outros aumento de curvatura corneana ${ }^{1}$. Outros estudos reportam aumento do astigmatismo, regular ou irregular e mudança no eixo. Estas mudanças são geralmente reversíveis após três meses, mas há casos em que não há reversão ${ }^{4}$. As alterações topográficas mais comumente observadas são o astigmatismo central irregular, perda da simetria radial e progressivo aplanamento do centro para a periferia ${ }^{1,10}$. O mecanismo pelo qual a topografia corneana é alterada após o uso de lentes de contato não é completamente conhecido, mas é provavelmente resultado de uma combinação de forças mecânicas e alterações fisiológicas, com conseqüente moldagem da córnea. Não foi possível estabelecer relação destas alterações com edema de córnea ${ }^{2}$. 
A redução na oferta de $\mathrm{O}_{2}$ e o acúmulo de $\mathrm{CO}_{2}$ sob a lente são relacionados ao edema crônico em alguns usuários de lente. Além disso, o lacrimejamento reflexo pode levar à hipotonia da lágrima, o que é um estímulo para o edema epitelial. A hipóxia crônica diminui a atividade metabólica aeróbica do epitélio, além de promover acúmulo de lactato no estroma. O edema de córnea acontece na região sob a lente e sua progressão pode levar à perda da transparência bem como às alterações na sua topografia, em função das variações da espessura corneana ${ }^{3}$.

A piora da visão corrigida com óculos é um dos parâmetros mais importantes no diagnóstico e seguimento destes pacientes, juntamente com a qualidade das miras ceratométricas. A recuperação da visão nestes casos é resultado da regularização e suavização da superfície óptica e da melhora na simetria central da córnea ${ }^{10}$. No caso apresentado, a refração, normalmente difícil de ser feita nos casos de ceratocone avançado, foi impossível de ser determinada no primeiro exame. Além disso a visão com lentes de contato foi de apenas 20/70. Estas alterações podem sugerir que uma eventual deformidade corneana já existisse antes da primeira adaptação. Após a suspensão da lente, a refração pode ser determinada, a visão com correção chegou a 20/200 e com as lentes 20/40.

A topografia corneana computadorizada é um método sensível na determinação de alterações corneanas não detectadas pela ceratometria. Usuários normais e assintomáticos podem apresentar alterações semelhantes àquelas dos portadores de deformidade corneana induzida por lente de contato ${ }^{5}$. As alterações topográficas no ceratocone em estágio inicial podem ser confundidas com "corneal warpage" e além disso, dependendo do alinhamento utilizado durante o exame, a descentração do ápice da córnea no ceratocone pode produzir erros significativos na topografia corneana computadorizada ${ }^{6,11}$.

No presente caso não houve dúvida quanto ao diagnóstico, já que o paciente apresentava ceratometria e biomicroscopia com dados considerados como patognomônicos de ceratocone ${ }^{11}$. Em relação ao exame, todas as topografias foram realizadas no mesmo aparelho (Eye Sys Technologies, Texas, USA). Todos os exames oftalmológicos foram feitos pelo autor.

Lews et al. ${ }^{7}$ em estudos com pacientes com ceratocone, referem casos de insucesso na adaptação de lente de contato por intolerância e/ou baixa de acuidade visual. Na verdade, como no caso apresentado é difícil saber se alguns de nossos casos de insucesso não são na realidade portadores de "Corneal Warpage". A exemplo deste paciente, alguns pacientes com ceratocone podem ter sido encaminhado para transplante de córnea não pela evolução da doença, mas sim pela alteração secundária ao uso das lentes.

A posição da lente no olho também tem um papel importante na fisiopatologia das deformidades. O posicionamento, quando livre das interferências das pálpebras, é determinada pela interação entre as forças do fluido lacrimal e a oposição da força da gravidade. A atração causada pela lágrima sob a lente depende da relação entre a sua curva base e a curvatura corneana. Além disso o ápice corneano deslocado pode contribuir para a descentração da lente ${ }^{12}$. O efeito mecânico da pálpebra superior pode ser a causa do aplanamento do eixo vertical em usuários de lentes rígidas já que a pressão exercida pode moldar parcialmente o eixo vertical da córnea ${ }^{3}$.

Wilson et al. ${ }^{1}$ não observaram correlação entre a curva base da lente usada e as topografias à época do diagnóstico e após a suspensão da lente. Porém, notou relação entre a topografia inicial e a posição da lente descentrada em relação ao centro anatômico da córnea. A descentração produz dois pontos de pressão focal sob a córnea, sendo um sobre a periferia e outro sobre a região central ${ }^{1}$. Portanto o mal posicionamento da lente pode ser considerado como um fator de risco e deve ser evitado. A dificuldade é que em muitos casos de ceratocone mesmo após vários testes inclusive com lentes especiais, a melhor lente possível não tem o posicionamento adequado apesar da boa visão e tolerância. Foi o que observamos em nosso paciente, já que na adaptação inicial a lente se encontrava em posição inferior. Além disso antes da primeira consulta, em que a ceratometria era maior que 60,00 OD nos dois meridianos, o paciente usava uma lente com curva base de $53,00 \mathrm{OD}$, o que pode ter causado uma maior pressão sobre a córnea. A exemplo de pacientes usuários de lentes que tem o uso das mesmas suspenso por apresentarem quadro topográfico sugestivo de ceratocone ${ }^{1}$, os usuários portadores desta patologia podem também se beneficiar da interrupção do uso das lentes quando houver suspeita de que a lente esteja alterando a anatomia da córnea.

A recuperação da visão e a estabilização da topografia são muito variáveis em casos normais, podendo chegar a cinco meses ou mais. Nos portadores de ceratocone esta melhora pode ser bastante imprevisível. No caso relatado houve melhora clínica evidente (refração e visão com lente de contato), além da estabilização da topografia, após seis meses de interrupção do uso da lente.

A apresentação deste caso é interessante por lembrar que a exemplo de qualquer paciente usuário de lente, os portadores de ceratocone também podem desenvolver "Corneal Warpage". Por isso, o oftalmologista deve estar atento para detectar o problema e fazer a distinção entre a própria evolução da doença e as alterações secundárias ao uso de lentes. Este quadro pode também explicar alguns casos de insucesso na adaptação de lente em portadores de ceratocone, por intolerância ou baixa de visão e até evitar que esses pacientes sejam submetidos precocemente a um transplante de córnea. Na realidade, para aqueles que não dedicam o tempo e a atenção necessários à adaptação de lente de contato, pode ser mais fácil e cômodo atribuir estas alterações à própria evolução do ceratocone.

\section{SUMMARY}

Introduction: Topographical alterations may occur as a secondary result of the use of hard or soft contact lenses. The expression corneal warpage is used to describe the corneal deformities caused by the lenses. Topographical map of initial keratoconus can be very similar to that of a patient with corneal warpage.

Purpose: To describe a contact lens wearer with keratoconus who developed corneal warpage symptoms that were diag- 
nosed and studied by topographies and a series of clinical examinations.

Case report: A forty-year-old patient with diagnosis of bilateral keratoconus for 12 years was wearing rigid gas permeable contact lenses since the initial diagnosis. On the first examination in 05/95, it was impossible to determine the RE refraction and keratometry was more than 60.00 D. Topography was carried out being compatible with keratoconus and a Soper RGP lens was fitted with a 20/70 vision. One year later a control topography showed an increase in the central curve and a flattening of the inferior periphery. The use of contact lenses was interrupted and after further six months there was a reversal of the changes, and a new Soper lens was fitted with a better pattern and a 20/40 vision.

Discussion: This case shows the occurrence of corneal deformity in a keratoconus patient using RGP lenses. The author discusses the pathophysiology and clinical and topographical diagnosis of corneal warpage, emphasizing the fact that patients with keratoconus as well as normal patients, can have these alterations which should be differentiated from the keratoconus evolution itself.

Keywords: Keratoconus; Corneal warpage; Contact lens.

\section{REFERÊNCIAS BIBLIOGRÁFICAS}

1. Wilson EW, Lin DTC, Klyce SD et al. Topographic changes in contact lens induced corneal warpage. Ophthalmology 1990;97:734-44.

2. Mobilia EF, Kenyon KR. Contact lens - induced corneal warpage. Int Ophthalmol Clin 1986;26:4353.

3. Sanaty M, Temel M. Corneal curvature changes in soft and rigid gas permeable contact lens wearers after two years of lens wear. CLAO J 1996;22:186-8.

4. Maeda N, Klyce S, Hamano H. Alteration of corneal asphericity in rigid gas permeable contact lens - induced warpage. CLAO J 1994;20:27-31.

5. Ruiz-Montenegro J, Mafra CH, Wilson S et al. Corneal topographic alterations in normal contact lens wearers. Ophtalmology 1993;100:128-34.

6. Smolek MK, Klyce SD, Maeda N. Keratoconus and contact lens - induced corneal warpage analysis using the keratomorph diagram. Invest Ophthalmol Vis Sc 1994;35:4192-203.

7. Lews MJ, Driche, Jr WT, Stern GA. The Clinical management of keratoconus: a 6 year retrospective study. CLAO J 1994;20:194-7.

8. Macsai MS, Varley GA, Krachmer JH. Development of keratoconus after contact lens wear. Patient characteristics. Arch Ophthalmol 1990;108:534-8.

9. Gasset AR, Houde WL, Garcia-Bengochea M. Hard contact lens wear as an enviroment risk in keratoconus. Am J Ophthalmol 1978;85:339-41.

10. Novo AG, Pavlopoulpos G, Feldman ST. Corneal topographic changes after refitting PMMA contact lens wearers into RGP materials. CLAO J 1995;21:47-51.

11. Chan JS, Mandell RB: Alignment effects in videokeratography of keratoconus. CLAO J 1997;23:23-8.

12. Carney LG, Mainstone JC, Carkeet A et all: Rigid lens dynamics: lid effects. CLAO J 1997;23:69-77.

\title{
VI CONGRESSO INTERNACIONAL DE CATARATA E CIRURGIA REFRATIVA
}

\author{
Ol a 4 de Abril de 2.000
}

Hotel Intercontinental - Rio de Janeiro - RJ

Promoção: Sociedade Brasileira de Catarata e Implantes Intra-Oculares

e Sociedade Brasileira de Cirurgia Refrativa

Informações: SBCCI/SBCR

R. Visconde de Pirajá, III - SI. 818

CEP 224 I0-003 - Rio de Janeiro - RJ

Tel./fax: (0xx2I) 523-7277 - home page: www.catarata-refrativa.com.br

LK Assessoria e Promoções

(0xx21) 580-9297 - Fax: (0xx21) 589-6751

e-mail:Ik@antares.com.br 